\title{
Carboxylic Sulfuric Anhydrides
}

\author{
C.J. Smith, Anna K. Huff, Rebecca B. Mackenzie, and Kenneth R. Leopold*
}

Department of Chemistry, University of Minnesota, 207 Pleasant St., SE,

Minneapolis, MN 55455 
Table S1. Observed transitions for the parent isotopologue, $\mathrm{HCCCOOSO}{ }_{2} \mathrm{OH}$

\begin{tabular}{|ccc|ccc|c|c|}
\hline $\mathrm{J}^{\prime}$ & $\mathrm{Ka}_{\mathrm{a}}{ }^{\prime}$ & $\mathrm{K}_{\mathrm{c}}{ }^{\prime}$ & $\mathrm{J}^{\prime \prime}$ & $\mathrm{Ka}_{\mathrm{a}}{ }^{\prime}$ & $\mathrm{K}_{\mathrm{c}}{ }^{\prime \prime}$ & $\begin{array}{c}\text { Observed Frequency } \\
{[\mathrm{MHz}]}\end{array}$ & $\begin{array}{c}\text { Obs-Calc } \\
{[\mathrm{MHz}]}\end{array}$ \\
\hline 3 & 1 & 3 & 2 & 1 & 2 & 5338.979 & 0.000 \\
3 & 0 & 3 & 2 & 0 & 2 & 5457.076 & 0.000 \\
3 & 1 & 2 & 2 & 1 & 1 & 5589.498 & 0.000 \\
2 & 1 & 2 & 1 & 0 & 1 & 5979.359 & -0.001 \\
2 & 1 & 1 & 1 & 0 & 1 & 6229.914 & -0.001 \\
4 & 1 & 4 & 3 & 1 & 3 & 7116.232 & 0.000 \\
4 & 0 & 4 & 3 & 0 & 3 & 7266.248 & 0.000 \\
4 & 1 & 3 & 3 & 1 & 2 & 7450.096 & 0.000 \\
3 & 1 & 3 & 2 & 0 & 2 & 7676.750 & 0.000 \\
3 & 1 & 2 & 2 & 0 & 2 & 8177.824 & 0.001 \\
5 & 1 & 5 & 4 & 1 & 4 & 8891.523 & 0.002 \\
5 & 0 & 5 & 4 & 0 & 4 & 9067.161 & -0.006 \\
5 & 1 & 4 & 4 & 1 & 3 & 9308.379 & -0.011 \\
4 & 1 & 4 & 3 & 0 & 3 & 9335.907 & 0.001 \\
4 & 1 & 3 & 3 & 0 & 3 & 10170.843 & 0.001 \\
6 & 1 & 6 & 5 & 1 & 5 & 10664.469 & -0.008 \\
6 & 0 & 6 & 5 & 0 & 5 & 10858.188 & -0.004 \\
6 & 2 & 5 & 5 & 2 & 4 & 10921.528 & -0.001 \\
6 & 2 & 4 & 5 & 2 & 3 & 10994.421 & 0.002 \\
6 & 1 & 5 & 5 & 1 & 4 & 11163.674 & -0.001 \\
7 & 1 & 7 & 6 & 1 & 6 & 12434.796 & -0.008 \\
7 & 0 & 7 & 6 & 0 & 6 & 12638.180 & -0.005 \\
7 & 2 & 6 & 6 & 2 & 5 & 12736.393 & -0.010 \\
7 & 2 & 5 & 6 & 2 & 4 & 12851.093 & 0.002 \\
7 & 1 & 6 & 6 & 1 & 5 & 13015.160 & 0.004 \\
8 & 1 & 8 & 7 & 1 & 7 & 14202.285 & -0.007 \\
8 & 0 & 8 & 7 & 0 & 7 & 14406.737 & -0.004 \\
8 & 2 & 7 & 7 & 2 & 6 & 14548.800 & -0.012 \\
8 & 2 & 6 & 7 & 2 & 5 & 14716.678 & 0.010 \\
8 & 1 & 7 & 7 & 1 & 6 & 14861.951 & 0.011 \\
\hline
\end{tabular}


Table S2. Observed transitions for the deuterated isotopologue, $\mathrm{HCCCOOSO}_{2} \mathrm{OD}$

\begin{tabular}{|ccc|ccc|c|c|}
\hline $\mathrm{J}^{\prime}$ & $\mathrm{Ka}^{\prime}$ & $\mathrm{Kc}^{\prime}$ & $\mathrm{J}^{\prime \prime}$ & $\mathrm{Ka}_{\mathrm{a}}^{\prime \prime}$ & $\mathrm{Kc}^{\prime \prime}$ & $\begin{array}{c}\text { Observed Frequency } \\
{[\mathrm{MHz}]}\end{array}$ & $\begin{array}{c}\text { Obs-Calc } \\
{[\mathrm{MHz}]}\end{array}$ \\
\hline 3 & 1 & 3 & 2 & 1 & 2 & 5312.485 & 0.002 \\
3 & 0 & 3 & 2 & 0 & 2 & 5434.772 & 0.001 \\
3 & 1 & 2 & 2 & 1 & 1 & 5572.986 & -0.001 \\
4 & 1 & 4 & 3 & 1 & 3 & 7080.640 & 0.000 \\
4 & 0 & 4 & 3 & 0 & 3 & 7235.413 & -0.002 \\
4 & 1 & 3 & 3 & 1 & 2 & 7427.793 & 0.002 \\
5 & 1 & 5 & 4 & 1 & 4 & 8846.628 & 0.001 \\
5 & 0 & 5 & 4 & 0 & 4 & 9026.923 & -0.001 \\
5 & 2 & 4 & 4 & 2 & 3 & 9068.412 & -0.001 \\
5 & 2 & 3 & 4 & 2 & 2 & 9115.160 & -0.002 \\
5 & 1 & 4 & 4 & 1 & 3 & 9280.024 & 0.000 \\
4 & 1 & 3 & 3 & 0 & 3 & 10091.278 & 0.000 \\
6 & 1 & 6 & 5 & 1 & 5 & 10610.043 & 0.000 \\
6 & 0 & 6 & 5 & 0 & 5 & 10807.541 & 0.001 \\
6 & 2 & 5 & 5 & 2 & 4 & 10877.759 & 0.003 \\
6 & 2 & 4 & 5 & 2 & 3 & 10958.596 & -0.001 \\
6 & 1 & 5 & 5 & 1 & 4 & 11128.884 & -0.002 \\
\hline
\end{tabular}


Table S3. Observed transitions for the ${ }^{34} \mathrm{~S}$ isotopologue, $\mathrm{HCCCOO}^{34} \mathrm{SO}_{2} \mathrm{OH}$

\begin{tabular}{|ccc|ccc|c|c|}
\hline $\mathrm{J}^{\prime}$ & $\mathrm{Ka}^{\prime}$ & $\mathrm{K}_{\mathrm{c}}$ & $\mathrm{J}^{\prime \prime}$ & $\mathrm{Ka}_{\mathrm{a}} \mathrm{K}_{\mathrm{c}}{ }^{\prime \prime}$ & $\begin{array}{c}\text { Observed Frequency } \\
{[\mathrm{MHz}]}\end{array}$ & $\begin{array}{c}\text { Obs-Calc } \\
{[\mathrm{MHz}]}\end{array}$ \\
\hline 3 & 1 & 3 & 2 & 1 & 2 & 5309.309 & 0.000 \\
3 & 0 & 3 & 2 & 0 & 2 & 5426.296 & 0.000 \\
3 & 1 & 2 & 2 & 1 & 1 & 5557.299 & -0.001 \\
4 & 1 & 4 & 3 & 1 & 3 & 7076.721 & -0.001 \\
4 & 0 & 4 & 3 & 0 & 3 & 7225.419 & 0.001 \\
4 & 1 & 3 & 3 & 1 & 2 & 7407.229 & 0.004 \\
5 & 1 & 5 & 4 & 1 & 4 & 8842.212 & 0.001 \\
5 & 0 & 5 & 4 & 0 & 4 & 9016.460 & 0.001 \\
5 & 1 & 4 & 4 & 1 & 3 & 9254.900 & 0.001 \\
6 & 1 & 6 & 5 & 1 & 5 & 10605.413 & -0.001 \\
6 & 0 & 6 & 5 & 0 & 5 & 10797.801 & -0.001 \\
6 & 2 & 5 & 5 & 2 & 4 & 10859.812 & 0.000 \\
6 & 1 & 5 & 5 & 1 & 4 & 11099.633 & -0.002 \\
\hline
\end{tabular}


Table S4. Cartesian coordinates for the minimum energy structure of the ${\mathrm{HCCCOOH}-\mathrm{SO}_{3}}_{3}$ van der Waals complex

\begin{tabular}{|cccc|}
\hline Atom & $\mathrm{X}$ & $\mathrm{Y}$ & $\mathrm{Z}$ \\
\hline $\mathrm{S} 1$ & -1.524935 & -0.120593 & 0.010886 \\
O2 & -1.936983 & -0.902187 & -1.087556 \\
O3 & -1.351654 & 1.293259 & -0.205351 \\
O4 & -1.758723 & -0.549727 & 1.335261 \\
C5 & 1.331926 & 0.20694 & -0.019515 \\
O6 & 0.408338 & -0.617268 & -0.056221 \\
O7 & 1.186549 & 1.487144 & 0.004286 \\
H8 & 0.211042 & 1.695993 & -0.045589 \\
C9 & 2.690392 & -0.265183 & -0.002718 \\
C10 & 3.810717 & -0.677057 & 0.010009 \\
C11 & 4.809486 & -1.044486 & 0.021394 \\
\hline
\end{tabular}


Table S5. Cartesian coordinates for the $\mathrm{HCCCOOH}-\mathrm{SO}_{3}$ optimized transition state geometry corresponding to $\pi_{2}+\pi_{2}+\sigma_{2}$ cycloaddition

\begin{tabular}{|cccc|}
\hline Atom & $\mathrm{X}$ & $\mathrm{Y}$ & $\mathrm{Z}$ \\
\hline $\mathrm{S} 1$ & -1.388945 & -0.164369 & 0.026165 \\
O2 & -1.987158 & -0.977559 & -0.953922 \\
O3 & -1.264521 & 1.263887 & -0.354695 \\
O4 & -1.607895 & -0.383682 & 1.403263 \\
C5 & 1.259123 & 0.259125 & -0.036403 \\
O6 & 0.328103 & -0.624786 & -0.128337 \\
O7 & 1.054097 & 1.486061 & 0.017538 \\
H8 & -0.11011 & 1.599081 & -0.131679 \\
C9 & 2.599952 & -0.251674 & -0.01013 \\
C10 & 3.714755 & -0.678055 & 0.014335 \\
H11 & 4.709245 & -1.056918 & 0.035457 \\
\hline
\end{tabular}


Table S6. Cartesian coordinates for the minimum energy structure of $\mathrm{HCCCOOSO} \mathrm{OH}_{2}$

\begin{tabular}{|cccc|}
\hline Atom & $\mathrm{X}$ & $\mathrm{Y}$ & $\mathrm{Z}$ \\
\hline $\mathrm{S} 1$ & -1.255346 & -0.19212 & 0.080457 \\
O2 & -1.982676 & -1.303303 & -0.372136 \\
O3 & -1.412263 & 0.940392 & -0.972667 \\
O4 & -1.357552 & 0.328595 & 1.387646 \\
C5 & 1.276042 & 0.33621 & -0.035812 \\
O6 & 0.309565 & -0.615994 & -0.137088 \\
O7 & 1.056736 & 1.508996 & 0.005119 \\
H8 & -0.880505 & 1.706494 & -0.689658 \\
C9 & 2.586006 & -0.266955 & -0.012862 \\
C10 & 3.679182 & -0.746791 & 0.008607 \\
H11 & 4.654056 & -1.172362 & 0.028826 \\
\hline
\end{tabular}




\section{S7. Vibrational Frequencies Used in the Calculation of Equilibrium Constants ${ }^{\mathrm{a}}$}

\begin{tabular}{|c|c|}
\hline & Vibrational Frequencies $\left[\mathrm{cm}^{-1}\right]$ \\
\hline $\mathrm{SO}_{3}$ & 506.6989, 527.4375, 527.4569, 1106.8185, 1422.3692, 1422.3789 \\
\hline $\mathrm{H}_{2} \mathrm{O}$ & $1574.4943,3770.1572,3867.9914$ \\
\hline $\mathrm{HCOOH}$ & $\begin{array}{l}\text { 627.5706, 653.6345, 1041.7412, 1130.1276, 1280.5455, 372.5985, } \\
\text { 1822.0771, 3006.5829, 3696.9804 }\end{array}$ \\
\hline $\mathrm{CF}_{3} \mathrm{COOH}$ & $\begin{array}{l}\text { 40.7012, 232.7321, 239.7646, 383.6738, 420.5047, 497.6585, 576.277, } \\
\text { 583.2998, 663.0726, 783.3429, 801.1812, 1145.9192, 1208.0186, } \\
\text { 1209.0662, 1272.9989, 1413.2803, 1874.8839, 3707.5728 }\end{array}$ \\
\hline $\mathrm{HCCCOOH}$ & $\begin{array}{l}\text { 105.9531, 236.9225, 523.994, 576.1024, 591.5254, 705.4713, 743.7378, } \\
\text { 765.7568, 814.9067, 1171.3332, 1338.7649, 1809.9133, 2203.3841, } \\
\text { 3373.5145, 3709.6874 }\end{array}$ \\
\hline cis $-\mathrm{H}_{2} \mathrm{C}=\mathrm{CHCOOH}$ & $\begin{array}{l}\text { 109.416, 275.4703, 473.4473, 485.6305, 615.077, 627.0953, 819.2523, } \\
\text { 827.9338, 1001.7772, 1005.3177, 1044.108, 1159.4701, 1256.3052, } \\
\text { 1336.2332, 1405.7725, 1674.2103, 1808.4874, 3077.3444, 3128.3276, } \\
\text { 3172.7924, 3718.1555 }\end{array}$ \\
\hline trans $-\mathrm{H}_{2} \mathrm{C}=\mathrm{CHCOOH}$ & $\begin{array}{l}\text { 106.9134, 285.8396, 474.3106, 523.412, 576.1121, 578.8281, 825.567, } \\
\text { 828.6128, 997.9554, 1011.7391, 1013.4851, 1190.6362, 1270.7 } \\
\text { 1343.159, 1412.6692, 1666.9741, 1807.983, 3080.3999, 3129.8505, } \\
\text { 3172.1813, 3726.9049 }\end{array}$ \\
\hline $\mathrm{C}_{6} \mathrm{H}_{5} \mathrm{COOH}$ & $\begin{array}{l}\text { 63.7872, 155.0351, 214.9714, 376.069, 401.7061, 421.3098, 486.552, } \\
\text { 571.2524, 608.2967, 627.9586, 681.0176, 713.9006, 761.8671, } \\
\text { 811.7348, 854.6088, 949.4845, 980.3887, 989.2642, 1002.7957, } \\
\text { 1025.2512, 1072.8976, 1104.7621, 1142.4466, 1160.5274, 1185.3497, } \\
\text { 1288.8681, 1305.6685, 1353.247, 1447.3661, 1491.0937, 1604.3315, } \\
\text { 1622.7906, 1801.9399, 3105.6102, 3118.6664, 3125.6407, 3134.8945, } \\
\text { 3142.0628, 3726.8952 }\end{array}$ \\
\hline $\mathrm{HCOOSO}_{2} \mathrm{OH}$ & $\begin{array}{l}\text { 115.1681, 210.199, 224.5259, 327.3168, 391.589, 454.4644, 501.1311, } \\
\text { 517.398, 543.7626, 704.8408, 798.0384, 898.9087, 1025.4064, } \\
\text { 1117.5564, 1178.0747, 1249.0399, 1360.4832, 1480.802, 1804.2194, } \\
\text { 3039.9509, 3593.9179 }\end{array}$ \\
\hline $\mathrm{CF}_{3} \mathrm{COOSO}_{2} \mathrm{OH}$ & $\begin{array}{l}\text { 33.659, 59.7617, 125.906, 130.0867, 213.4388, 240.9383, 301.7476, } \\
\text { 339.3254, 388.0873, 411.9105, 443.3191, 487.4153, 516.1564, 538.544, } \\
\text { 540.29, 593.6691, 727.4515, 757.7737, 783.0713, 863.1836, 910.0831, } \\
\text { 1128.9636, 1165.2222, 1225.013, 1254.4137, 1272.9407, 1348.1933, } \\
\text { 1492.7524, 1859.3445, 3617.7411 }\end{array}$ \\
\hline $\mathrm{HCCCOOSO}_{2} \mathrm{OH}$ & $\begin{array}{l}\text { 62.7881, 118.9511, 124.7129, 226.0294, 246.7874, 328.6845, 379.1342, } \\
\text { 411.765, 455.124, 492.8376, 539.6013, 596.8604, 619.9755, 720.6421, } \\
\text { 746.803, 763.4579, 788.222, 880.3429, 926.059, 1179.0156, 1188.8611, } \\
\text { 1256.4507, 1480.9475, 1795.3924, 2207.914, 3368.8391, 3591.6384 }\end{array}$ \\
\hline s-trans $\mathrm{H}_{2} \mathrm{C}=\mathrm{COOSO}_{2} \mathrm{OH}$ & $\begin{array}{l}\text { 54.611, 110.3278, 139.5927, 165.2104, 254.4213, 332.7197, 377.8441, } \\
\text { 460.3911, 472.681, 488.9382, 525.0998, 543.9566, 563.4051, 637.6198, } \\
\text { 786.3402, 826.1199, 872.4665, 918.7452, 1001.2534, 1007.9076, } \\
\text { 1017.3845, 1189.7535, 1224.3922, 1266.6066, 1273.707, 1407.7028, } \\
\text { 1471.6646, 1662.483, 1793.9665, 3085.9289, 3133.9245, 3178.4572, } \\
\text { 3569.8813 }\end{array}$ \\
\hline s-cis $\mathrm{H}_{2} \mathrm{C}=\mathrm{COOSO}_{2} \mathrm{OH}$ & $\begin{array}{l}\text { 60.6056, 100.589, 132.9482, 156.7229, 287.1394, 327.7048, 359.773, } \\
\text { 391.007, 464.824, 488.5592, 527.5345, 547.5747, 587.9946, 682.6181, }\end{array}$ \\
\hline
\end{tabular}




\begin{tabular}{|l|l|}
\hline & $776.4365,817.0795,871.4771,918.396,997.7614,1016.8607$, \\
& $1060.0354,1148.0241,1190.0348,1250.3494,1294.7075,1400.1368$, \\
& $1473.9538,1666.4018,1792.5309,3081.7773,3140.1422,3177.7491$, \\
& 3555.3507 \\
\hline $\mathrm{C}_{6} \mathrm{H}_{5} \mathrm{COOSO}$ & $\mathrm{OH}$ \\
& $41.5257,47.5203,113.393,123.4034,165.1328,228.1149,292.1349$, \\
& $344.2336,385.0124,400.1832,430.3017,449.0615,485.4753,506.728$, \\
& $544.0051,579.9145,607.3655,675.9251,677.2055,709.4192,782.596$, \\
& $813.7524,835.3058,854.1723,901.6926,953.9271,979.3023,991.9123$ \\
& $1008.8679,1024.9796,1063.8378,1088.3788,1146.9377,1167.5987$, \\
& $1192.227,1232.9379,1271.3208,1293.7278,1310.5185,1449.6553$, \\
& $1470.4909,1490.2013,1604.0114,1620.3656,1782.0937,3110.111$, \\
& $3123.2448,3129.5692,3137.9597,3144.8564,3555.6223$ \\
\hline $\mathrm{SO}_{3}-\mathrm{H}_{2} \mathrm{O}$ & $41.7197,189.7902,200.305,205.9795,494.118,506.2818,507.0287$, \\
& $560.2235,602.8162,1102.405,1403.6676,1421.5156,1558.0916$, \\
\hline & $3709.765,3806.1054$ \\
\hline $\left.\mathrm{H}_{2} \mathrm{O}\right)_{2}-\mathrm{SO}_{3}$ & $17.557162 .6302,207.6576,240.1332,286.4604,293.2407,334.0971$, \\
& $357.5905,512.1988,526.9913,544.4125,555.9943,569.7683$, \\
& $797.8347,1088.3691,1130.3895,1363.2186,1419.1391,1570.0905$, \\
& $1577.2879,2922.9689,3659.8682,3738.2539,3811.2076$ \\
\hline
\end{tabular}

(a) The values in the table were obtained from the M06-2X/6-311++G(3df,3pd) level of theory and were scaled by a factor of 0.97 . 
Table S8. Rotational Constants and Dissociation Energies Used in the Calculations of Equilibrium Constants

\begin{tabular}{|c|c|c|}
\hline & $A, B, C[\mathrm{MHz}]$ & $D_{e}$ (hartree) $^{\mathrm{a}}$ \\
\hline $\mathrm{SO}_{3}$ & 10543.626, 10543.626, 5271.713 & 623.229702 \\
\hline $\mathrm{H}_{2} \mathrm{O}$ & $829677.55,433104.86,284560.02$ & 76.377668 \\
\hline $\mathrm{HCOOH}$ & 78320.692, $12236.909,10581.709$ & 189.599512 \\
\hline $\mathrm{CF}_{3} \mathrm{COOH}$ & $3903.273,2518.972,2088.704$ & 526.420135 \\
\hline $\mathrm{HCCCOOH}$ & 12277.865, 4161.592, 3108.099 & 265.632706 \\
\hline cis $-\mathrm{H}_{2} \mathrm{C}=\mathrm{CHCOOH}$ & 11240.206, 4293.414, 3106.736 & 266.896944 \\
\hline trans $-\mathrm{H}_{2} \mathrm{C}=\mathrm{CHCOOH}$ & $10860.909,4430.007,3146.567$ & 266.896565 \\
\hline $\mathrm{C}_{6} \mathrm{H}_{5} \mathrm{COOH}$ & 3919.808, 1236.906, 940.218 & 420.340862 \\
\hline $\mathrm{HCOOSO}_{2} \mathrm{OH}$ & $4541.770,2026.889,1926.781$ & 812.858739 \\
\hline $\mathrm{CF}_{3} \mathrm{COOSO}_{2} \mathrm{OH}$ & 2158.913, 591.945, 560.621 & 1149.675633 \\
\hline $\mathrm{HCCCOOSO}_{2} \mathrm{OH}$ & $3389.781,957.114,873.140$ & 888.891850 \\
\hline$s$-trans $\mathrm{H}_{2} \mathrm{C}=\mathrm{COOSO}_{2} \mathrm{OH}$ & 2874.374, 1057.195, 909.673 & 890.159595 \\
\hline$s$-cis $\mathrm{H}_{2} \mathrm{C}=\mathrm{COOSO}_{2} \mathrm{OH}$ & $3667.119,901.388,842.265$ & 890.159437 \\
\hline $\mathrm{C}_{6} \mathrm{H}_{5} \mathrm{COOSO}_{2} \mathrm{OH}$ & 2054.544, 384.145, 345.444 & 1043.604363 \\
\hline $\mathrm{SO}_{3}-\mathrm{H}_{2} \mathrm{O}$ & 5215.536, 3975.785, 3939.703 & 699.622709 \\
\hline$\left(\mathrm{H}_{2} \mathrm{O}\right)_{2}-\mathrm{SO}_{3}$ & 4612.963, 2011.670, 1922.849 & 776.021545 \\
\hline
\end{tabular}

(a) Obtained at the CCSD(T)/CBS(D-T)//M06-2X/6-311++G(3df,3pd) level of theory. 